\title{
Distribution and Histopathological Evaluation of Eyelid, Conjunctival and Orbital Tumours
}

\author{
${ }^{1}$ A.V.Subba Rao, ${ }^{2}$ M. L. Karthika, ${ }^{3}$ R V Manasa \\ ${ }^{\text {I }}$ Associate Professor, Dept. of Ophthalmology, Dr PSIMS \& RF, India) \\ ${ }^{2}$ (Senior Resident, Rangaraya Medical College, Kakinada, India) \\ ${ }^{3}$ (Statistician, Dept. of Community Medicine, GIMSAR, Vishakapatnam, India)
}

\begin{abstract}
:
Introduction: Ocular neoplasm being the most important cause of morbidity and mortality, early diagnosis and detection of the pathology is crucial for proper treatment and better prognosis. In clinical practice, eye Tumours and Tumour-like lesions can be classified into extra- and intra-ocular. Benign growth of eyelid includes xanthelasma, molluscum, warts, naevus, angioma and other tumers common to skin and cutaneous glands. Present study conducted with following Objectives: 1. To highlight the benign and malignant Tumours of eyelid, conjunctiva and orbit for early diagnosis and management. 2. Histopathological analysis to establish the type and subtype of benign and malignant Tumours.

Methodology: This present study was a hospital based, longitudinal study conducted between August 2012 to August 2014. Patients with benign and malignant Tumours of eyelid, conjunctiva and orbit were included in the study and those who had Infective, inflammatory and traumatic lesions of eyelids, conjunctiva and orbit were excluded from study.

Results: The study comprised 36 cases, out of them 19 subject had Eyelid, 9 Conjunctival and 8 Orbital Tumours. Among 19 eyelid Tumours, we found 17 Benign Tumours and 2 Malignant Tumours. Most common benign Tumour of eyelid seen in our study was Nevus (7 cases) followed by epidermoid cyst (5 cases), Papilloma(3 cases), xanthelasma(1 case), keratin cyst(1 case). Most common benign tumour of conjunctiva seen in our study was Nevus (4 cases), followed by dermolipoma (2 cases), papilloma (1 case) and dermoid cyst (1 case).All 4 Nevus cases were melanocytic nevus type. Most common benign Tumour of orbit in our study was Hemangioma(3 cases), followed by neurofibroma(2 cases) and Dermoid cyst( 2 cases).

Conclusion: The clinical evaluation and histopathological examination of the ocular Tumours has immense role in classifying the Tumours, establishing the final diagnosis and also in planning the most effective treatment modalities to reduce the morbidity and mortality to the possible extent.
\end{abstract}

Keywords: Benign tumour of eyelid, conjunctiva and orbit. Malignant tumour of eyelid, conjunctiva and orbit.

\section{Introduction}

Ocular neoplasm being the most important cause of morbidity and mortality, early diagnosis and detection of the pathology is crucial for proper treatment and better prognosis. These Tumours arise as primaries from orbital and ocular tissues or spread from contiguous anatomic structures like the paranasal sinuses, nasopharynx, brain, and as secondaries from distant structures. The histopathological characteristics of these Tumours are critical to their biologic behaviour, line of management, outcome, and prognosis. Peri-ocular area is a common site for benign and malignant lesions in all age groups. Peri-ocular structures that include the eyelids, eyebrows, lacrimal sac, and conjunctiva are derived from the surface ectoderm and congenital anomalies can present as Tumour or Tumour-like lesion at birth or soon after birth. In clinical practice, eye Tumours and Tumour-like lesions can be classified into extra- and intra-ocular. Benign growth of eyelid include xanthelasma, molluscum, warts, naevus, angioma and other tumers common to skin and cutaneous glands. Xanthelasma are slightly raised yellow plaques most commonly found in upper and lower lids and oftenly seen in elderly women having diabetes and hypercholesterolaemia.Orbital tumors are rare and can occure at all stages in life. Benign growths include dermoid cyst, dermolipoma angioma, osteoma, plexiform neuroma, meningioma and meningoencephalocele ${ }^{1}$. Basal cell carcinoma is the most common malignant eyelid tumour predominantly effecting lower lid and medial canthal area ${ }^{2}$. Orbital tumours are not very common. These includes primary, secondary and meta static tumours. Orbit is mainly (90\%) involved by malignant non- Hodgkin B-cell lymphomas in case of malignant orbital Lymphomas ${ }^{3 .}$ In our study, only extra- ocular Tumours that is eyelid, conjunctiva and orbital Tumours were investigated. So the present study conducted with following Objectives: 1. To highlight the benign and malignant Tumours of eyelid, conjunctiva and orbit for early diagnosis and management. 2. Histopathological analysis to establish the type and subtype of benign and malignant Tumours. 


\section{Materials and Methods}

A Total number of 36 patients coming to Pinnamaneni Siddhartha Institute of Medical Sciences and Research Foundation fulfilling the study criteria were included in the study.

Inclusion Criteria: Patients with benign and malignant Tumours of eyelid, conjunctiva and orbit who have given informed consent.

Exclusion criteria: Infective, inflammatory and traumatic lesions of eyelids, conjunctiva and orbit. This present study was a hospital based, longitudinal study conducted between August 2012 to August 2014. In each case clinical diagnosis was confirmed by histopathology. Each case was analyzed based on Sex and age distribution of lesions, site of lesions, clinical diagnosis of Tumours and Histopathological result of Tumours. If the lesion had clinical features of benign Tumour, lesion was excised and sent in specimen bottle soaked in formalin for histopathological examination. If the eyelid lesion had clinical features of malignancy, wide surgical excision along with atleast $4 \mathrm{~mm}$ normal tissue around it. Specimen excised was labelled and placed in a bottle containing formalin and sent for histopathology. In case of histopathologically confirmed malignant lesion, margin clearance was noted.

All samples were sent to Department of Pathology, for histopathological examination. In pathology department macroscopic examination of tissues was done. Resected margins of received biopsy was fixed in $10 \%$ formalin and processed conventionally by embedding in paraffin blocks with later sectioning. Sections were stained with Haematoxylin and Eosin and special stains as and when required. Section was studied for histopathological type and subtype of Tumour.

\section{Results}

The study comprised 36 cases in which we found 19 Eyelid, 9 Conjunctival and 8 Orbital Tumours. 1) Eyelid Tumours:

A) Distribution of types of Eyelid Tumours: Among 19 eyelid Tumours we found 17 Benign Tumours and 2 Malignant Tumours.

B) Age, Sex and Site Distribution of Eyelid Tumours: Mean age of patients was 52yrs with range between 090yrs. Benign Tumours were more frequent in middle aged patients seen most commonly in $4^{\text {th }}$ and $5^{\text {th }}$ decade. Malignant Tumours were seen in elderly patients in $6^{\text {th }}$ and 9 th decade. Related to gender, 11 cases were male with 10 benign and 1 malignant Tumour and 7 cases were female with 6 benign Tumours and 1 malignant Tumour. In our study eyelid Tumours were seen more predominantly in male patients and equal sex prediliction of malignant Tumours. The topographical analysis of lesions showed the frequency of locations: 11 cases at upper lid and 8 cases at lower lid. Benign Tumours most commonly in upperlid and malignant Tumours equal distribution.

C) Types of Benign Tumours of Eyelid: Most common benign Tumour of eyelid seen in our study was Nevus (7 cases) followed by epidermoid cyst (5 cases), Papilloma( 3 cases), xanthelasma( 1 case), keratin cyst( 1 case). Among Nevus cases 4 were compound nevus type and 3 were junctional nevus type.

D) Types of Malignant Tumours of Eyelid: Only malignant Tumour of eyelid seen in our study was sebaceous gland carcinoma.

\section{2) Conjunctival Tumours:}

A) Distribution of Different Types of Conjunctival Tumours: Among 9 conjunctival Tumours we found 8 benign and 1 malignant Tumour.

B) Age, Sex and Site Distribution of Conjunctival Tumours: Mean age of patients with Conjunctival tumours was 27 yrs with range between $0-70 y r s$. Benign and malignant Tumours were most commonly seen in $1^{\text {st }}$ and $2^{\text {nd }}$ decades. Related to gender, overall 7 cases were male with 6 benign and 1 malignant Tumour and 2 cases were female and 2 were benign. In our study Conjunctival tumours were predominantly seen in male patients.

C) Types of Benign Tumours of Conjunctiva: Most common benign tumour of conjunctiva seen in our study was Nevus ( 4 cases), followed by dermolipoma ( 2 cases), papilloma ( 1 case) and dermoid cyst (1 case).All 4 Nevus cases were melanocytic nevus type.

D) Types of Malignant Tumours of Conjunctiva: Only malignant tumour of Conjunctiva seen in our study was Squamous Intraepithelial Neoplasia.

\section{3) Orbital Tumours:}

A) Distribution of different types of Orbital Tumours: Among 8 Orbital Tumours, we found 7 benign and 1 malignant tumour.

B) Age, Sex and Site distribution of Conjunctival Tumours: Mean age of patients with orbital Tumours was $24 \mathrm{yrs}$ with range between $0-70 \mathrm{yrs}$. Benign Tumours were more frequent in $1^{\text {st }}$ and $2^{\text {nd }}$ decades and malignant Tumour in $6^{\text {th }}$ decade. Related to gender, overall 5 cases were female with 4 benign and 1 malignant Tumour 
and 3 cases were male with 3 cases benign. In our study orbital Tumours were seen more predominantly in female patients.

C) Types of Orbital Benign Tomours: Most common benign Tumour of orbit in our study was Hemangioma(3 cases), followed by neurofibroma(2 cases) and Dermoid cyst(2 cases). Among Hemongioma cases 2 were capillary Hemangioma and 1 was intramuscular Hemangioma.

D) Types of Orbital Malignant Tomours: Only malignant Tumour of orbit seen in our study was NonHodgkins Lymphoma.

\section{Discussion}

This was the hospital based longitudinal study which included 36 patients of Eyelid, Conjunctiva and Orbit Tumours attending our hospital. Among 36 Tumours Eyelid Tumours are 19, Conjunctival Tumours are 9 and Orbital Tumours are 8 in number. Eyelid Tumours represented 53\%, Conjunctival Tumours 25\% and Orbital Tumours $22 \%$ of all Tumours.

Among the eyelid Tumours we studied benign Tumours are identified in 17 cases and malignant Tumours in 2 cases. Benign lesions represented $89 \%$ and malignant $11 \%$ of all eyelid Tumours. Deprez M et al found that benign Tumours largely predominated over malignant Tumours representing $84 \%$ cases $^{1}$. Another study conducted by Paul S et al also found that benign lesions were more common which were seen in $75.9 \%$ of all cases among 855 cases studied and malignant lesions were seen in $24.1 \%^{3}$. Topographical analysis of eyelid lesions showed that most common location of eyelid Tumour is upper eyelid representing 57.8\% followed by lower eyelid representing $42.1 \%$. Coroi $\mathrm{M} \mathrm{C}$ et al found that Tumours developed mostly in lower eyelid than upper eyelid ${ }^{2}$. In this study, among benign Tumours of eyelid common location is upper eyelid in 10 cases representing $59 \%$ followed by lower eyelid in 7cases representing 41\%. Study conducted by Asadi Amoli F et al studied 1103 eyelid Tumours showed that most common benign Tumour was Nevus (37.7\%) followed by Hamartoma $(19.6 \%)$ and papilloma $(15.9 \%)^{8}$. Another recent study also showed that most common benign Tumour was Melanocytic Nevi (35\%) among 182 patients studied in a $10 \mathrm{yr}$ survey from a tertiary hospital in Tehran $^{5}$. The most prevalent benign masses in the studies by Coroi ${ }^{2}$ et a and Deprez ${ }^{1}$ et al were papilloma. Some of these disparities are interesting due to demographic differences. In our study most common benign Tumour was Nevus representing $41 \%$ of all benign cases. Different studies have reported various frequencies of benign Tumours some of which are similar to our study Apart from Nevus, we found 5cases of Epidermoid cyst, 3cases of papilloma and 1 each case of xanthelasma and keratin cyst was seen. In our study most common malignant lesions seen were SGC (100\%). Studies from other countries have reported difference in types and frequiences of malignant eyelid Tumours. In most of studies from other countries BCC is the most frequent malignant Tumour of the eyelid ${ }^{1,7,11}$. However, frequency of SGC is higher in Asian countries ${ }^{36}$. In our study SGC was most common malignant Tumour consistent with studies from Asian countries. Studies from Singapore reported incidence of $10-40 \%$ of $S_{G C}^{6}$. A study from central India by Jahagirdar SS et al concluded that SGC is almost as common as BCC in India ${ }^{4}$.

For the Conjunctival Tumours, we identified Benign Tumours in 8 cases and Malignant Tumour in 1 case. Benign lesions represented $89 \%$ and malignant $11 \%$. Zaidi $\mathrm{N}$ et al found that benign Tumours were more frequent which were seen in $74.50 \%$ of all cases studied and malignant Tumours were seen in $25.50 \%{ }^{14}$. Another study conducted by Bastola et al also found that benign lesions were more common which were seen in $79 \%$ of all cases studied and malignant lesions were seen in $21 \%{ }^{13}$. In our study Benign lesions were represented by Nevus (4 cases), followed by dermolipoma ( 2 cases), papilloma (1 case) and dermoid cyst (1 case). Bastola et al found that most common benign lesions were granuloma pyogenicum( $22.5 \%)$ followed by intradermal nevus $(4.5 \%)$. In our study most common malignant lesion seen was squamous intraepithelial neoplasia consistent with other studies ${ }^{12,13,14}$.

For the Orbital Tumours we studied we identified benign Tumours in 7 cases and malignant Tumour in 1 case. Benign lesions represented $88 \%$ and malignant $12 \%$. Radha. J.et al found that benign lesions were more frequent which were seen in $70.8 \%$ and malignant lesions were seen in $29.2 \%{ }^{15}$. Another study conducted by Nath $\mathrm{K}$ et al found benign lesions were most frequent which were seen in $85.81 \%$ and malignant lesions were seen in $14.17 \%^{16}$. In our study Benign lesions were represented by Hemangioma(3 cases), followed by neurofibroma(2 cases) and Dermoid cyst(2 cases). Radha. J.et al also found that among benign lesions hemangiomas were most common $(23.5 \%)^{15}$. In our study, the mean age of patients with benign orbital Tumours was $18 \mathrm{yrs}$ and they were more prevalent in females representing $57 \%$. Nath $\mathrm{K}$ et al found that age of the patients having primary orbital tumours varied from I to 69 years although they were more common during the first two decades $(69 \text { cases })^{16}$. Our study also showed more prevalence in first two decades. In our study malignant lesion seen was Non-Hodgkin Lymphoma consistent with other studies. Study conducted by J H Koopman et al found that most common tumour type was lymphoma representing $67 \%$ of all malignant Tumours ${ }^{17}$. 


\section{Conclusion}

Ocular Tumours though rare in clinical practice, should be evaluated and the diagnosis must be confirmed by doing histopathological examination. This study revealed that Benign ocular Tumours are the commonest involving the eyelid, conjunctiva and orbit. The histopathological evaluation helped in the final diagnosis of the Tumours. Hence our study enlightened the diagnosis of malignant and benign Tumours. This helped immensely in carrying out effective treatment modalities, which in turn enabling to explain mortality and morbidity to the patients. To conclude the clinical evaluation and histopathological examination of the ocular Tumours has immense role in classifying the Tumours, establishing the final diagnosis and also in planning the most effective treatment modalities to reduce the morbidity and mortality to the possible extent.

\section{References}

a. Parson M. Diseases of the Lids, In:Parson's Diseases of the Eye, $20^{\text {th }}$ Ed, Noida, Elsevier, 2008 , p 439.

b. Nema HV, Nema N. Diseases of the Lids, In: Text book of Opthalmology, $6^{\text {th }}$ Ed, New Delhi, Jaypee publisher, 2012 , p 429.

c. $\quad$ Khurana AK. Diseases of the Orbit, In: Comprehensive Opthalmology, $5^{\text {th }}$ Ed, New Delhi, New Age International Publishers, $p 424$.

[2]. Deprez M, Uffer S. Clinicopathological features of eyelid skin Tumours. A retrospective study of 5504 cases and review of literature. American Journal of Dermatopathology, May 2009;31(3):256-262.

[3]. Paul S, Vo DT, Wilkiss RZ. Malignant and Benign eyelid lesions in San Francisco:study of a diverse urban population. American Journal of Clinical Medicine, Winter 2011;8(1):40-46.

[4]. Coroi MC, Rosca E, Mutiu G, Coroi T, Bonta M. Eyelid Tumours: Histopathological and Clinical study performed in Country Hospital of Oradea between 2000-2007. Romanian journal of Morphology and Embryology 2010;51(1):111-115.

[5]. Asadi Amoli F, Mohammadi S Z, Haeri H, Kasaei. A study of eyelid Tumour Tehran Farabi hospital (1988-98). Tehran University Medical Journal 2003;61(2):127-135.

[6]. Abbas B, Mehdi T, Azadeh K, Reza BZ,Hamed E, Maryam A, Hossein S. Eyelid Masses: A 10-year Survey from a Tertiary Eye Hospital in Tehran. Middle East African Journal of Ophthalmology 2013jul-Sep;20(3):187-192.

[7]. Chang CH, Chang SM, Lai YH, Huang J, Su MY, Wang HZ, et al. Eyelid Tumours in southern Taiwan: A 5-year survey from a medical university.Kaohsiung J Med Sci. 2003;19:549-54.

[8]. Xu XL, Li B, Sun XL, Li LQ, Ren RJ, Gao F. Clinical and pathological analysis of 2639cases of eyelid Tumours. Zhonghua Yan ke Za Zhi, 2008 Jan;44(1):38-41.

[9]. Pornpanich K, Chindasub P. Eyelid Tumours in Siriraj Hospital from 2000-2004. J Med Assoc Thai. 2005;88:S11-4.

[10]. Lee SB, Saw SM, Au Eong KG, Chan TK, Lee Hp. Incidence of eyelid cancers in Singapore from 1968 to 1995. British Journal of Ophthalmology, May 1999;83(5):595-597.

[11]. Jahagirdar SS, Thakre TP, kale SM, Kulkarni H, Mamtani M.A clinicopathological study of eyelid malignancies from Central India. Indian Journal of Ophthalmology 2007;55(2): 109-112.

[12]. N Zaidi, K Nath, R Gogi. Primary conjunctival tumours. Indian J Ophthalmol 1980;28:171-8.

[13]. P Bastola , S Koirala , G Pokhrel , P Ghimire , RK Adhikari. A Clinico-histopathological study of orbital and ocular lesions; a multicenter study, Journal of Chitwan Medical College 2013; 3(4).

[14]. Laila Hassan Mohamad Elshazly. A Clinicopathologic Study of Excised Conjunctival Lesions; Middle East Afr J Ophthalmol. 2011 Jan-Mar; 18(1): 48-54

[15]. Radha. J , Ani Sreedhar. Orbital Tumours - A Clinico Pathological Study; KSOS Journal September 2005;261-265.

[16]. Nath K, Gogi R. Primary orbital tumours.Indian J Ophthalmol 1977;25:10-16

[17]. JH Koopman, M van der Heiden-van der Loo2. Incidence of primary malignant orbital tumours; 2011; 25:461-465. 